\title{
Under-use of appropriate blood pressure-lowering and lipid-lowering therapy in the Busselton baby boomer population
}

Wai Yiu, Matthew Knuiman, Hilary Wallace, Joseph Hung

\section{Background and objective \\ Data are sparse on how well the absolute risk approach is implemented in primary healthcare. The aim of this study was to quantify absolute cardiovascular disease (CVD) risk, appropriate use of blood pressure (BP)- lowering and lipid-lowering therapy, and clinical target responses in the Busselton baby boomer population.}

\section{Methods}

This was a cross-sectional study of 5107 people aged $45-69$ years (54.6\% female) who participated in the 2010-2015 Busselton Healthy Ageing Study.

\section{Results}

Overall, $16.1 \%$ of participants had prior CVD (5.8\%) or a high primary CVD risk (10.3\%). The frequency of use of a guideline-recommended combination of BP-lowering and lipid-lowering therapy was $46.2 \%$ in participants with prior CVD, compared with only $16.8 \%$ in those with high primary CVD risk ( $P<0.001)$. Among the high-risk participants who were receiving recommended combination therapy, only $42.7 \%$ achieved target systolic BP levels and $42.1 \%$ achieved target total cholesterol levels.

\section{Discussion}

These data confirm substantial under-treatment of Australian adults who are at high CVD risk. Enhanced implementation of absolute CVD risk assessment and evidence-based treatment in high-risk adults has potential for substantial health gains.
CARDIOVASCULAR DISEASE (CVD) is the leading cause of morbidity and mortality in Australia. ${ }^{1,2}$ It is largely preventable through lifestyle modifications and pharmacological therapy directed at adults who have a high absolute CVD risk. ${ }^{3,4}$ An individual's absolute CVD risk is calculated on the basis of the combined effect of multiple risk factors, and individuals at highest absolute risk will obtain the highest absolute benefit from pharmacological treatments. ${ }^{3-5}$

The Australian National Vascular Disease Prevention Alliance (NVDPA) promotes the routine use of absolute risk assessment and provides guidance on clinical management to reduce the risk of cardiovascular events in adults aged $\geq 45$ years (or $\geq 35$ years for Aboriginal and Torres Strait Islander people) with no previous history of CVD. ${ }^{3}$ According to NVDPA guidelines, asymptomatic people clinically determined as high risk or calculated to have $>15 \%$ absolute risk of a primary CVD event within the next five years are recommended to be on a combination of lipid-lowering and blood pressure (BP)-lowering medications. ${ }^{3,4}$

Data are sparse on how well the NVDPA guidelines are being implemented in primary healthcare. In the Australian Health Survey conducted in 2011-2012, Banks et al reported that approximately one-fifth of Australians aged 45-74 years had a high absolute risk of CVD, but only $44.2 \%$ with existing CVD and $24.3 \%$ at high primary CVD risk received guideline-recommended BP-lowering and lipid-lowering therapy. ${ }^{6}$
This study used data from the Busselton Healthy Ageing Study, which was based on a rural population, ${ }^{7}$ in comparison to the Australian Health Survey, which sampled populations mainly in major urban areas. ${ }^{8}$ The aims of this study were to quantify absolute CVD risk in the Busselton baby boomer population, ascertain use of guideline-recommended BP-lowering and lipid-lowering therapy in this rural population, and determine the proportion of participants at high CVD risk taking combination therapy who achieved recommended target $\mathrm{BP}$ and cholesterol levels.

\section{Methods}

\section{Study population}

The Busselton Healthy Ageing Study included baby boomers who were surveyed in the City of Busselton in Western Australia between 2010 and 2015 when they were aged 45-69 years. ${ }^{7}$ Of the 8223 baby boomers on the electoral roll, $82 \%$ were eligible and able to be contacted, and 5107 (76\%) participated in the study. All participants provided informed written consent, and this study received ethics approval from the University of Western Australia Human Ethics Committee and the University of Notre Dame Human Research Ethics Committee (RA/4/1/2203).

\section{Data and variables}

The study protocol has been previously described. ${ }^{7}$ All participants completed a 
self-administered health questionnaire. Height, weight and a single resting systolic $\mathrm{BP}(\mathrm{SBP})$ and diastolic BP (DBP) were measured; 12-lead electrocardiogram recorded; and fasting blood samples assayed for total cholesterol, high-density lipoprotein cholesterol and estimated glomerular filtration rate. ${ }^{7}$ There were 5003 participants in the final analysis after excluding 104 (2\%) with missing data.

\section{Cardiovascular disease risk assessment} The NVDPA guidelines recommend that adults with certain clinical features be classified as high risk without the need to calculate absolute risk (Appendix 1, available online only). ${ }^{3}$ For the remaining participants, the five-year absolute CVD risk was calculated using the Framingham CVD risk equation. ${ }^{3}$ The five-year absolute CVD risk was then categorised as low $(<10 \%)$, moderate (10-15\%) or high (>15\%). ${ }^{3}$ The calculated absolute high-risk group and the clinically-determined high-risk group together form the 'high primary CVD risk' category.

\section{Statistical analysis}

The proportions of participants in separate CVD risk categories were calculated according to age groups and sex. The researchers calculated the proportion of people in each risk group taking BP-lowering and/or lipid-lowering medications and, within the high CVD risk groups, the proportions taking medications who reached recommended target BP and cholesterol levels. Differences in proportions across groups were assessed using the chi-square test, and the significance threshold was set at $P<0.05$.

\section{Results}

\section{Clinical risk characteristics}

The sample comprised 5003 participants aged 45-69 years, of which $54.6 \%$ were female and $94.2 \%$ had no prior CVD history. The participant distribution of CVD risk factors included in the NVDPA algorithm is shown in Table 1. As expected, CVD risk factors were more frequent in people with existing CVD.

\section{Absolute cardiovascular disease risk categories}

Among people without prior CVD, $11.0 \%(n=517)$ were assessed as being at high primary CVD risk on the basis of absolute risk calculator $(n=190)$ or clinical determinants $(\mathrm{n}=327) ; 10.6 \%$ and $78.4 \%$ were positioned in moderate and low absolute risk categories, respectively (Table 2). Among those categorised as having a high primary CVD risk, prevalence increased with age and was higher in males when compared with females (Table 2). Participants with high primary risk and those with established CVD comprised $16.1 \%(n=807)$ of the total cohort $(21.7 \%$ of men and $11.5 \%$ of women).

\section{Use of blood pressure-lowering and lipid-lowering therapy}

The use of combination BP-lowering and lipid-lowering medication was higher in people with prior CVD when compared with those determined at high primary CVD risk (46.2\%, compared with $16.8 \%, P<0.001$; Table 3$)$. Within the high-primary CVD risk group, people clinically determined at high risk were more likely to be on combination therapy than those at high calculator-derived risk (22.6\%, compared with $6.8 \%, P<0.001)$. Overall, the use of combination therapy among the high-primary risk group rose with increasing age and body mass index category (both trend $P<0.001$ ), but there was no significant difference between the two sexes or trend with income or education level (data not shown).

\section{Clinical response to treatment}

As shown in Table 4, there was no significant difference in reaching SBP targets between participants at high CVD risk who took both BP-lowering and lipid-lowering medications and those who did not (41.6\%, compared with $42.7 \%$, $P=0.78)$. Similar results were seen with DBP targets (69.7\%, compared with $69.5 \%, P=0.96)$. However, sex-specific results were discordant, with a higher proportion of men compared with women on combination therapy achieving SBP targets $(P<0.001)$. Participants receiving combination therapy were significantly more likely to achieve total cholesterol targets than those who were not receiving combination therapy (42.1\%, compared with $6.7 \%, P<0.001)$, and this finding was consistent for low-density lipoprotein cholesterol (LDL-C) targets and in both sexes (Table 4). However, women receiving combination therapy were less likely to reach their total cholesterol target than men $(28.4 \%$, compared with $49.0 \%, P<0.001)$, with similar results for the LDL-C target. In results stratified by age group as well as sex (not shown), the percentages of the high-risk group reaching targets were very similar across age groups $45-54$ years, $55-64$ years and 65-69 years.

\section{Discussion}

Busselton baby boomers had a similar CVD risk factor distribution to that reported in the Australian Health Survey sample aged 45-74 years, ${ }^{6}$ and consequently there was a similar proportion (approximately $10 \%$ ) who were predicted to have a high absolute risk of a primary CVD event within five years. Among the high-primary CVD risk group, less than one-fifth were taking the recommended combination of BP-lowering and lipid-lowering therapy, and more than half were taking neither medication type - findings that are similar to those reported by Banks et al. ${ }^{6}$ Even among those with high CVD risk who were taking combination therapy, less than half achieved recommended individual risk factor targets.

This study provides further evidence regarding the under-use of guidelinerecommended therapy among adult Australians at high primary CVD risk. The use of combination therapy was much higher in patients with prior CVD or clinically determined at high risk when compared with those who had a calculated high absolute risk. This may reflect that clinicians more readily identify high-risk individuals on the basis of clinical criteria than on absolute risk calculation. In addition, those at calculated moderate absolute risk had greater use of combination therapy, compared with those at high absolute risk $(10.2 \%$, compared with $6.8 \%$ ). This could be due to physicians assessing individual risk 
Table 1. Clinical risk characteristics of the Busselton population sample according to age group and prior cardiovascular disease status

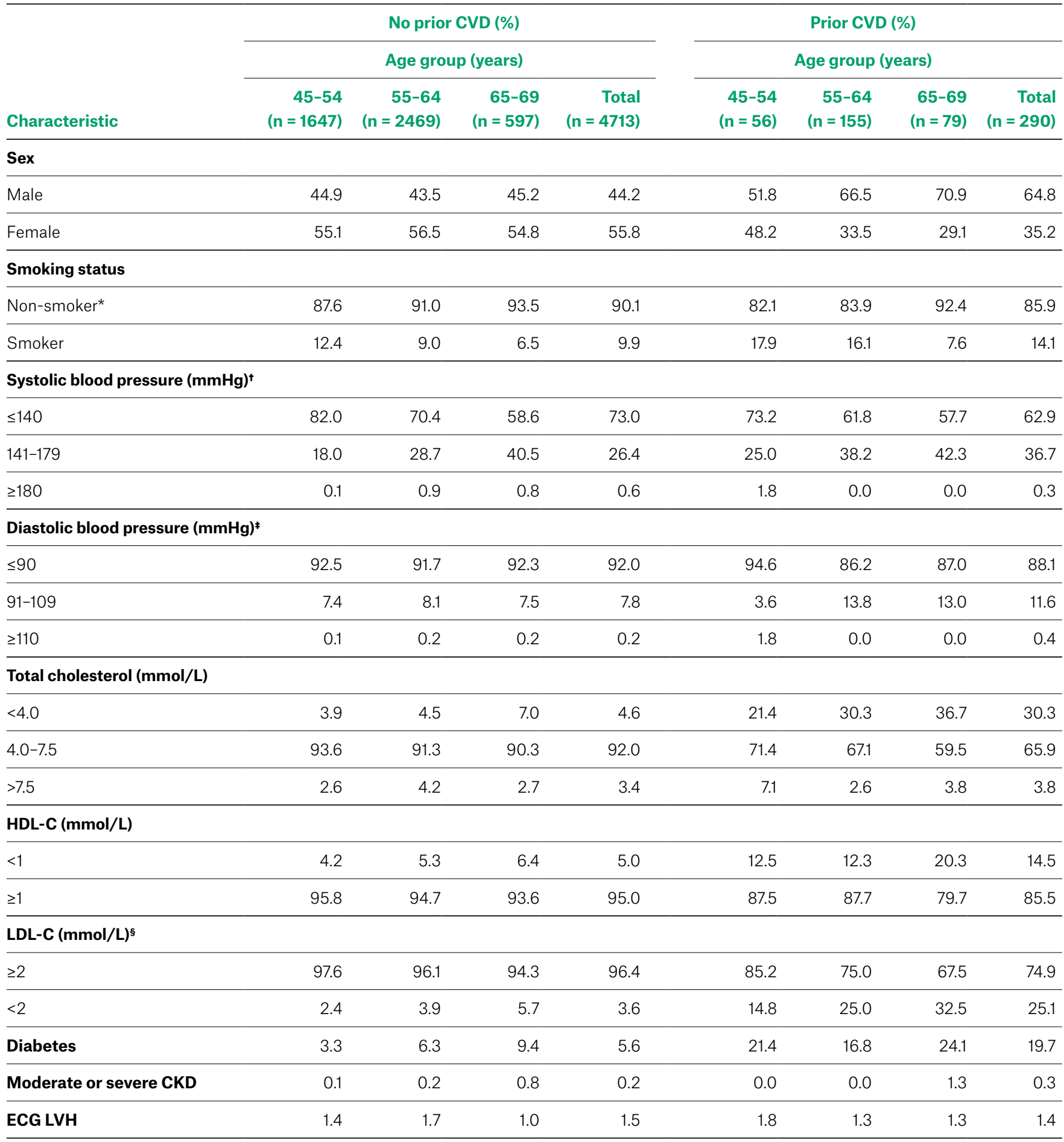

*Non-smoker category includes ex-smokers and never-smokers

${ }^{\dagger}$ Missing data for systolic blood pressure $(n=4)$

*Missing data for diastolic blood pressure $(n=5)$

${ }^{s}$ Missing data for $L D L(n=57)$

CVD, cardiovascular disease; BMI, body mass index; HDL-C, high-density lipoprotein cholesterol; LDL-C, low-density lipoprotein cholesterol; CKD, chronic kidney disease defined as estimated glomerular filtration rate $<45 \mathrm{~mL} / \mathrm{min} / 1.73 \mathrm{~m}^{2} ; E C G L V H$, left ventricular hypertrophy on electrocardiography 
Table 2. Cardiovascular risk categories of the Busselton population sample stratified by age group and gender*

\begin{tabular}{|c|c|c|c|c|c|c|c|}
\hline \multirow[t]{4}{*}{ Age group (years) } & \multicolumn{5}{|c|}{ No prior CVD $(n=4713)$} & $\begin{array}{r}\text { Prior CVD } \\
(\mathrm{n}=290)\end{array}$ & $\begin{array}{r}\text { Total } \\
(n=5003)\end{array}$ \\
\hline & \multicolumn{3}{|c|}{$\begin{array}{l}\text { Not clinically at high CVD risk }(n=4386) \\
\text { by Framingham risk category }{ }^{\dagger}\end{array}$} & \multirow[t]{2}{*}{$\begin{array}{r}\text { Clinical } \\
\text { high risk } \\
(\mathrm{n}=327)\end{array}$} & \multirow[t]{2}{*}{$\begin{array}{r}\text { High primary } \\
\text { CVD risk } \\
(\mathrm{n}=517)\end{array}$} & & \\
\hline & $\begin{array}{r}\text { Low } \\
(<10 \%)\end{array}$ & $\begin{array}{r}\text { Moderate } \\
(10-15 \%)\end{array}$ & $\begin{array}{r}\text { High } \\
(>15 \%)\end{array}$ & & & & \\
\hline & $\%(n)$ & $\%(n)$ & $\%(n)$ & $\%(n)$ & $\%(n)$ & $\%(n)$ & $\%(n)$ \\
\hline
\end{tabular}

\section{Both sexes}

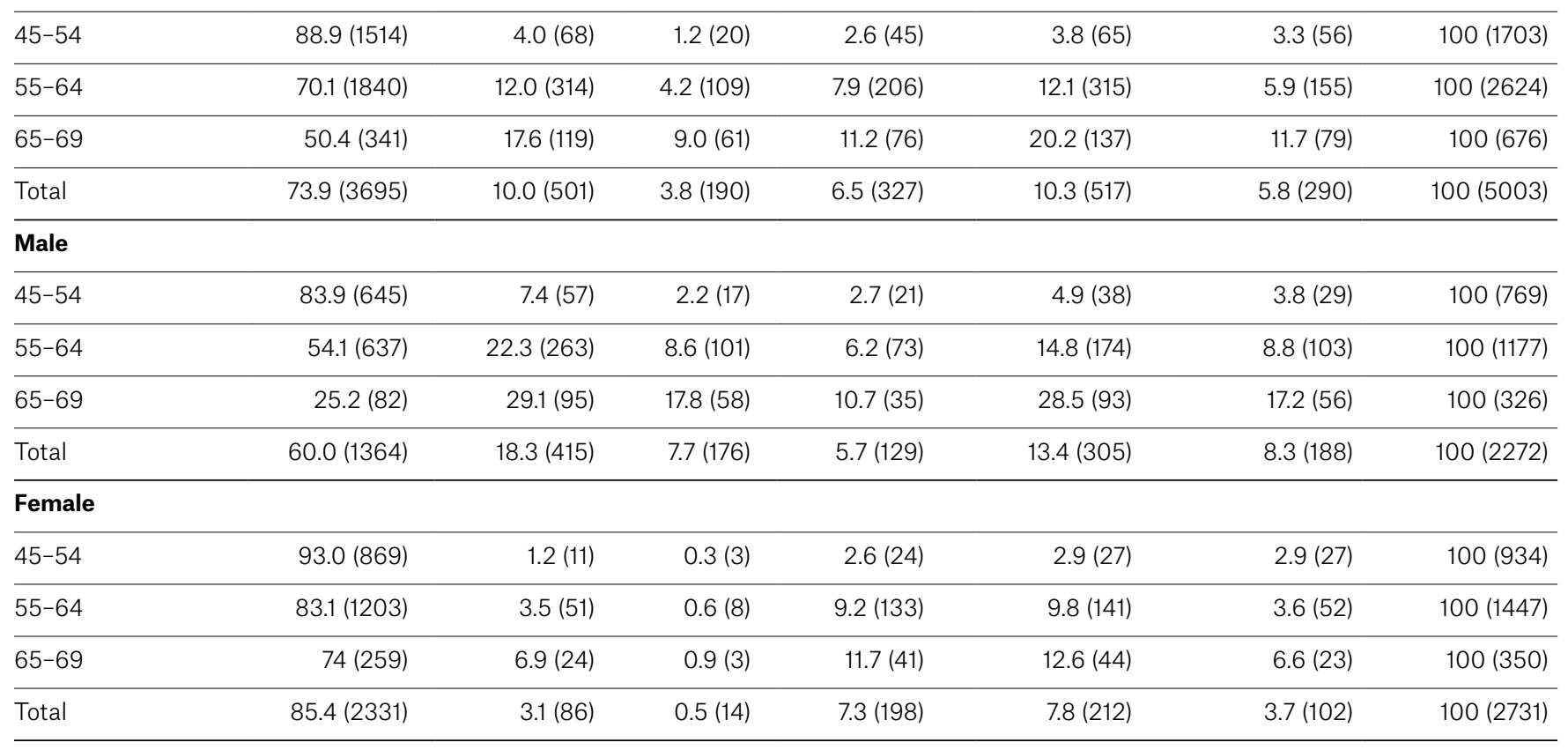

${ }^{*}$ Categories are not all mutually exclusive so the sum of percentages is greater than $100 \%$.

${ }^{\dagger}$ Estimated five-year absolute CVD risk using the Framingham CVD risk equation

\#Individuals clinically determined at high risk of CVD include adults with diabetes and aged $>60$ years, diabetes with microalbuminuria, moderate or severe

chronic kidney disease, systolic blood pressure $\geq 180 \mathrm{mmHg}$ or diastolic blood pressure $\geq 110 \mathrm{mmHg}$, or serum total cholesterol $>7.5 \mathrm{mmol} / \mathrm{L}$.

${ }^{\S}$ Combined Framingham high risk and clinical high risk of CVD

CVD, cardiovascular disease

factors (eg considering cholesterol level alone) and not using tools that quantify overall CVD risk. This approach can lead to substantial misclassification, with both under- and overestimation of absolute risk and consequent under- or overtreatment.

Current evidence suggests that less than half of the patients attending primary care in Australia have their CVD risk assessed and documented..$^{9-11}$ Potential barriers to GPs conducting routine absolute risk assessment include limited consultation time, uncertainty about risk factors not included in the absolute risk tools and preference for clinical judgement. ${ }^{12}$ Possible ways to improve CVD risk assessment include having a built-in absolute risk tool within the prescribing system, and better education and incentives for GPs to implement NVDPA guidelines. In Australian primary health settings, an electronic decision support tool was shown to improve CVD risk measurements but did not increase prescription rates for high-risk patients. ${ }^{13}$

The NVDPA guidelines have well-established goals for BP and cholesterol levels for people with high CVD risk. ${ }^{3}$ Among the high-risk Busselton participants, less than half who were taking the guideline-recommended combination therapy achieved target SBP and cholesterol targets. These findings are concordant with an Australian registry survey of patients at high CVD risk in which half the subjects failed to achieve the BP target, and $24 \%$ did not attain the total cholesterol target despite widespread use of BP-lowering and lipid-lowering therapy. ${ }^{14}$

Achieving target $\mathrm{BP}$ control is difficult, often requiring more than one medication, ${ }^{15}$ and there is frequent reluctance to intensify therapy or add additional medications because of potential side-effects associated with 'aggressive' BP 
Table 3. Proportion and number of individuals in the Busselton population sample who were receiving blood pressure lowering and/or lipid-lowering medications, according to their cardiovascular disease risk category and age group*

\begin{tabular}{|c|c|c|c|c|c|c|}
\hline & \multicolumn{5}{|c|}{ No prior CVD $(n=4713)$} & \multirow[t]{2}{*}{$\begin{array}{r}\text { Prior CVD } \\
(n=290)\end{array}$} \\
\hline & \multicolumn{3}{|c|}{$\begin{array}{l}\text { Not clinically at high CVD risk }(n=4386) \\
\text { by Framingham risk category }{ }^{+}\end{array}$} & \multirow[t]{2}{*}{$\begin{array}{r}\text { Clinical high } \\
\text { risk }^{*} \\
(n=327)\end{array}$} & \multirow[t]{2}{*}{$\begin{array}{r}\text { High primary } \\
\text { CVD risk } \\
(n=517)\end{array}$} & \\
\hline & $\begin{array}{r}\text { Low }(<10 \%) \\
(n=3695)\end{array}$ & $\begin{array}{r}\text { Moderate } \\
(10-15 \%) \\
(n=501)\end{array}$ & $\begin{array}{r}\text { High (>15\%) } \\
(n=190)\end{array}$ & & & \\
\hline & $\%(n)$ & $\%(n)$ & $\%(n)$ & $\%(n)$ & $\%(n)$ & $\%(n)$ \\
\hline All age groups $(n=5003)$ & $100.0(3695)$ & $100.0(501)$ & $100.0(190)$ & $100.0(327)$ & $100.0(517)$ & $100.0(290)$ \\
\hline Lipid-lowering medication & $12(442)$ & $19.4(97)$ & $15.8(30)$ & $32.7(107)$ & $26.5(137)$ & $60.0(174)$ \\
\hline BP-lowering medication & $17.2(636)$ & $29.3(147)$ & $31.1(59)$ & $38.5(126)$ & $35.8(185)$ & $61.4(178)$ \\
\hline Taking neither medication & $75.9(2803)$ & $61.5(308)$ & $60.0(114)$ & $51.4(168)$ & $54.5(282)$ & $24.8(72)$ \\
\hline $45-54$ years $(n=1703)$ & $100.0(1514)$ & $100.0(68)$ & $100.0(20)$ & $100.0(45)$ & $100.0(65)$ & $100.0(56)$ \\
\hline Lipid-lowering medication & $5.9(90)$ & $23.5(16)$ & $10.0(2)$ & $0.0(0)$ & $3.1(2)$ & $42.9(24)$ \\
\hline BP-lowering medication & $10.0(152)$ & $22.1(15)$ & $20(4)$ & $6.7(3)$ & $10.8(7)$ & $39.3(22)$ \\
\hline $\begin{array}{l}\text { BP-lowering and lipid-lowering } \\
\text { medication }\end{array}$ & $1.7(26)$ & $16.2(11)$ & $0.0(0)$ & $0.0(0)$ & $0.0(0)$ & $23.2(13)$ \\
\hline Taking one medication only & $12.5(190)$ & $13.2(9)$ & $30(6)$ & $6.7(3)$ & $13.8(9)$ & $35.7(20)$ \\
\hline Taking one medication only & $22.3(411)$ & $30.3(95)$ & $32.1(35)$ & $25.2(52)$ & $27.6(87)$ & 25.2 (39) \\
\hline Taking neither medication & 71.5 (1316) & $61.8(194)$ & $59.6(65)$ & $51.9(107)$ & $54.6(172)$ & $26.5(41)$ \\
\hline $65-69$ years $(n=676)$ & $100.0(341)$ & $100.0(119)$ & $100.0(61)$ & $100.0(76)$ & $100.0(137)$ & $100.0(79)$ \\
\hline Lipid-lowering medication & $25.5(87)$ & $26.1(31)$ & $16.4(10)$ & $47.4(36)$ & $33.6(46)$ & $73.4(58)$ \\
\hline BP-lowering medication & $32.8(112)$ & $31.1(37)$ & $32.8(20)$ & $63.2(48)$ & $49.6(68)$ & 74.7 (59) \\
\hline $\begin{array}{l}\text { BP-lowering and lipid-lowering } \\
\text { medication }\end{array}$ & $13.8(47)$ & $12.6(15)$ & $6.6(4)$ & $35.5(27)$ & $22.6(31)$ & $58.2(46)$ \\
\hline Taking one medication only & $30.8(105)$ & $31.9(38)$ & $36.1(22)$ & $39.5(30)$ & $38.0(52)$ & $31.6(25)$ \\
\hline Taking neither medication & $55.4(189)$ & $55.5(66)$ & $57.4(35)$ & $25.0(19)$ & $39.4(54)$ & $10.1(8)$ \\
\hline $\begin{array}{l}{ }^{*} \text { Categories are not all mutually exclusiv } \\
{ }^{+} \text {Estimated five-year absolute CVD risk } \\
\text { Individuals clinically determined at high } \\
\text { chronic kidney disease, systolic blood pr } \\
{ }^{8} \text { Combined Framingham high risk and cl } \\
\text { CVD, cardiovascular disease; BP, blood }\end{array}$ & $\begin{array}{l}\text { the sum of perc } \\
\text { the Framinghar } \\
\text { of CVD include } \\
\text { re } \geq 180 \mathrm{mmHg} \\
\text { high risk of } \mathrm{CV} \\
\text { ure }\end{array}$ & $\begin{array}{l}\text { os is greater th } \\
\text { risk equation } \\
\text { with diabetes } \\
\text { tolic blood pre }\end{array}$ & $\begin{array}{l}100 \% \text {. } \\
\text { d aged }>60 \text { year } \\
\text { ire } \geq 110 \mathrm{mmHg} \text {, }\end{array}$ & $\begin{array}{l}\text { liabetes with micr } \\
\text { serum total choles }\end{array}$ & $\begin{array}{l}\text { lbuminuria, moder } \\
\text { rol >7.5 mmol/L. }\end{array}$ & severe \\
\hline
\end{tabular}


Table 4. Proportion and number of individuals in the Busselton population sample with prior CVD or in the high primary CVD risk group reaching their blood pressure and cholesterol targets according to their treatment group and sex

\begin{tabular}{|c|c|c|c|c|}
\hline $\begin{array}{l}\text { Individuals with prior CVD or } \\
\text { in high primary CVD risk group } \\
(\mathrm{n}=\mathbf{8 0 7})\end{array}$ & $\begin{array}{l}\text { SBP target } \\
\text { achieved* }\end{array}$ & $\begin{array}{l}\text { DBP target } \\
\text { achieved* }^{*}\end{array}$ & $\begin{array}{r}\text { Total cholesterol } \\
\text { target achieved } \\
(<4.0 \mathrm{mmol} / \mathrm{L})\end{array}$ & $\begin{array}{r}\text { LDL-C target } \\
\text { achieved } \\
(<2.0 \mathrm{mmol} / \mathrm{L})^{+}\end{array}$ \\
\hline
\end{tabular}

\section{Both sexes}

BP-lowering and lipid-lowering

medication $(n=221)$

$41.6(92)$

$69.7(154)$

$42.1(93)$

$36.9(79)$

Taking neither or one medication

only $(n=586)$

42.7 (250)

69.5 (407)

6.7 (39)

5.8 (32)

$P$ value

0.778

0.956

$<0.001$

$<0.001$

\section{Male}

BP-lowering and lipid-lowering

medication $(n=147)$

$44.9(66)$

$68.0(100)$

$49.0(72)$

$40.8(58)$

Taking neither or one medication

only $(n=346)$

35.8 (124)

63.6 (220)

$9.2(32)$

8.0 (26)

$P$ value

0.058

0.349

$<0.001$

$<0.001$

\section{Female}

BP-lowering and lipid-lowering

medication $(n=74)$

$35.1(26)$

$73.0(54)$

$28.4(21)$

$29.2(21)$

Taking neither or one medication

only $(n=240)$

$52.5(126)$

0.009

77.9 (187)

0.383

$2.9(7)$
$<0.001$

$2.6(6)$

$P$ value

*Missing data for systolic/diastolic BP $(n=4)$. For patients without diabetes, SBP target is $\leq 140 \mathrm{mmHg}$ and DBP target is $\leq 90 \mathrm{mmHg}$. For patients with diabetes, SBP target is $\leq 130 \mathrm{mmHg}$ and $D B P$ target is $\leq 80 \mathrm{mmHg}$.

${ }^{\dagger} M i s s i n g$ data for $L D L-C$ target $(n=39)$

CVD, cardiovascular disease; SBP, systolic blood pressure; DBP, diastolic blood pressure; LDL-C, low-density lipoprotein cholesterol; BP, blood pressure

control, especially in elderly patients. ${ }^{16,17}$ Furthermore, poor patient adherence occurs because of medication adverse effects, complicated dosing regimens and a negative impact on quality of life from multiple medications. ${ }^{18,19}$ It is also important to recognise that clinical inertia related to an interplay of clinician, patient and practice system factors remains a major contributor to inadequate chronic disease management; ultimately, a better understanding of clinical inertia and the development of specific interventions to reduce it is crucial. ${ }^{20}$

Among the high-risk participants receiving recommended combination therapy, women were less likely to achieve SBP and total cholesterol targets when compared with men $(28.4 \%$, compared with $49.0 \%$ ). Women attending primary healthcare services in Australia are known to be less likely than men to have CVD risk factors measured and absolute risk assessed. ${ }^{21}$ Further, younger women with high CVD risk are less likely to be prescribed preventive medications, possibly because of a misconception that these women are protected from CVD. ${ }^{21}$ Women are also more likely to stop taking a statin as a result of new or worsening muscle symptoms ${ }^{22}$ and less likely to tolerate high-intensity statins to achieve their LDL-C goals. ${ }^{23}$ Better communication between healthcare providers and patients regarding benefits and risks of statin therapy may help reduce the current sex difference in lipid goal attainment. ${ }^{22}$

Although achieving individual risk factor targets is an ideal goal, it is likely that interventions that drive even a moderate increase in uptake of guideline-recommended therapy in people at high CVD risk will have a greater impact on CVD prevention overall. The goal is to reduce the patient's level of absolute risk, which is achieved by managing several risk factors, as the evidence shows that moderate reduction in several risk factors is more effective in reducing overall CVD risk than a major reduction in one risk factor. ${ }^{3,24}$ In this regard, polypill, or fixed-dose combination therapy, has been advocated to improve prevention by enhancing medication adherence and access, although further trial data are required.$^{25}$

\section{Limitations}

For participants who were already on BP-lowering and/or lipid-lowering medication at the time of data collection, 
it was not possible to calculate their pre-treatment absolute CVD risk. As a result, the CVD risk of people on treatment may have been underestimated. Although there was a high participation rate (76\%), the population sample was drawn from the City of Busselton, confined to a relatively narrow age range of people in a regional setting, and very homogenous (>99\% Caucasian); therefore, generalisability of these findings to the wider Australian population, specific ethnic populations, ages and subgroups may be limited. Despite limitations in the study design, the CVD risk profile of this cohort was consistent with the Banks et al study, ${ }^{6}$ which is a representative study of Australia.

\section{Conclusion}

These findings show the considerable and continuing gap between clinical practice and evidence-based guidelines for cardiovascular prevention in Australia. There are major opportunities to reduce the burden of CVD with routine absolute risk assessment, lifestyle modifications and pharmacological therapy where appropriate. However, further studies are needed to explore and define how best to address the evidence-treatment gap in light of the known interplay between patient, logistic and clinician factors that determine compliance with guidelines in the real-world environment.

\section{Authors}

Wai Yiu BPharm, MD, medical student, School of Medicine, The University of Notre Dame, WA Matthew Knuiman PhD, Professor, Faculty of Health and Medical Sciences, School of Population and Global Health, The University of Western Australia, WA Hilary Wallace BVMS, PhD, Lecturer Research, School of Medicine, The University of Notre Dame, WA Joseph Hung MB, BS, FRACP, Emeritus Professor, Faculty of Health and Medical Sciences, Medical School, The University of Western Australia, WA. joe.hung@uwa.edu.au

Competing interests: None.

Funding: The Busselton Healthy Ageing Study was conducted under the management of the Busselton Population Medical Research Institute, which receives funding from the Office of Science and Department of Health of the Government of Western Australia, the City of Busselton, a bequest of the late Dr Janet Elder and private donors. We (co-authors) had full access to all relevant data in this study, and supporting sources had no involvement in data analysis and interpretation, or in the writing of the article.

Provenance and peer review: Not commissioned, externally peer reviewed.

\section{Acknowledgement}

The Busselton Investigators wish to acknowledge the National Health and Medical Research Council (NHMRC) Equipment Grant scheme for purchase of essential equipment. We thank the Western Australian Country Health Service - South West for core infrastructure support. We thank the operational team in Busselton for participant recruitment and data collection, and the community of Busselton for their ongoing support and participation.

\section{References}

1. Australian Bureau of Statistics. 3303.0 Causes of death, Australia 2016. Belconnen, ACT: ABS, 2017. Available at www.abs. gov.au/ausstats/abs@.nsf/Lookup/ by\%20Subject/3303.0 2016 Main\%20 Features Australia\%27s\%20leading $\% 20$ causes\%20of\%20death,\%202016 3 [Accessed 5 October 2019].

2. Australian Institute of Health and Welfare. Australian burden of disease study: Impact and causes of illness and death in Australian 2011. Australian Burden of Disease Study series no. 3. BOD 4. Canberra: AlHW, 2016.

3. National Vascular Disease Prevention Alliance. Guidelines for the management of absolute cardiovascular disease risk. Melbourne: NVDPA, 2012.

4. Jackson R, Lawes CM, Bennett DA, Milne RJ, Rodgers A. Treatment with drugs to lower blood pressure and blood cholesterol based on an individual's absolute cardiovascular risk. Lancet 2005;365(9457):434-41. doi: 10.1016/S01406736(05)17833-7.

5. Goff DC Jr, Lloyd-Jones DM, Bennett G, et al. 2013 ACC/AHA Guideline on the assessment of cardiovascular risk: A report of the American College of Cardiology/American Heart Association Task Force on practice guidelines. Circulation 2014;129(25 Suppl 2):S49-S73. doi: 10.1161/01. cir.0000437741.48606.98.

6. Banks E, Crouch SR, Korda RJ, et al. Absolute risk of cardiovascular disease events, and blood pressure- and lipid-lowering therapy in Australia. Med J Aust 2016;204(8):320. doi: 10.5694/ mja15.01004.

7. James A, Hunter M, Straker L, et al. Rationale, design and methods for a community-based study of clustering and cumulative effects of chronic disease processes and their effects on ageing: the Busselton healthy ageing study. BMC Public Health 2013;13:936. doi: 10.1186/1471-2458-13-936.

8. Australian Bureau of Statistics. 4363.0.55.001 - Australian health survey: Users' guide, 2011-13. Belconnen, ACT: ABS, 2013. Available at www.abs.gov.au/AUSSTATS/ abs@.nsf/DetailsPage/4363.0.55.0012011 13?OpenDocument [Accessed 4 October 2019].

9. Heeley EL, Peiris DP, Patel AA, et al. Cardiovascular risk perception and evidence - Practice gaps in Australian general practice (the AusHEART study). Med J Aust 2010;192(5):254-59. doi: 10.5694/ j.1326-5377.2010.tb03502.x.

10. Webster RJ, Heeley EL, Peiris DP, Bayram C, Cass A, Patel AA. Gaps in cardiovascular disease risk management in Australian general practice. Med J Aust 2009;191(6):324-29. doi: 10.5694/ j.1326-5377.2009.tb02816.x.

11. Sheppard JP, Fletcher K, McManus RJ, Mant J. Missed opportunities in prevention of cardiovascular disease in primary care: A cross-sectional study. Br J Gen Pract 2014;64(618):e38-46. doi: 10.3399/ bjgp14X676447.

12. Bonner C, Jansen J, McKinn S, et al. General practitioners' use of different cardiovascular risk assessment strategies: A qualitative study. Med J Aust 2013;199(7):485-89.
13. Peiris D, Usherwood T, Panaretto K, et al. Effect of a computer-guided, quality improvement program for cardiovascular disease risk management in primary health care: The treatment of cardiovascular risk using electronic decision support cluster-randomized trial. Circ Cardiovasc Qual Outcomes 2015;8(1):87-95. doi: 10.1161/ CIRCOUTCOMES.114.001235.

14. Reid CP, Nelson MR, Shiel L, Chew D, Connor G, DeLooze F. Australians at risk: Management of cardiovascular risk factors in the REACH registry. Heart Lung Circ 2007;17(2):114-18. doi: 10.1016/j. hlc.2007.07.009.

15. Taddei S. Combination therapy in hypertension: What are the best options according to clinical pharmacology principles and controlled clinical trial evidence? Am J Cardiovasc Drugs 2015;15(3):185-94. doi: 10.1007/s40256-0150116-5.

16. Elliott WJ. What factors contribute to the inadequate control of elevated blood pressure? J Clin Hypertens (Greenwich) 2008;10(1 Suppl 1):20-26.

17. Chowdhury EK, Owen A, Krum H, et al. Barriers to achieving blood pressure treatment targets in elderly hypertensive individuals. J Hum Hypertens 2013;27(9):545-51. doi: 10.1038/jhh.2013.11.

18. Wright JT Jr, Cushman W, Oparil S, et al. A randomized trial of intensive versus standard blood-pressure control. N Engl J Med 2015;373(22):2103-116. doi: 10.1056/NEJMoa1511939.

19. National Heart Foundation of Australia. Guideline for the diagnosis and management of hypertension in adults - 2016. Melbourne: National Heart Foundation of Australia, 2016.

20. O'Connor PJ, Sperl-Hillen JM, Johnson PE, Rush WA, Blitz G. Clinical inertia and outpatient medical errors. In: Henriksen K, Battles JB, Marks ES, Lewin DI, editor. Rockville, MD: Agency for Healthcare Research and Quality (US), 2005.

21. Hyun KK, Redfern J, Patel A, et al. Gender inequalities in cardiovascular risk factor assessment and management in primary healthcare. Heart 2017;103(7):492-98. doi: 10.1136/ heartjnl-2016-310216.

22. Karalis DG, Wild RA, Maki KC, et al. Gender differences in side effects and attitudes regarding statin use in the Understanding Statin Use in America and Gaps in Patient Education (USAGE) study. J Clin Lipidol 2016;10(4):833-41. doi: 10.1016/j.jacl.2016.02.016.

23. Virani SS, Woodard LD, Ramsey DJ, et al. Gender disparities in evidence-based statin therapy in patients with cardiovascular disease. Am J Cardiol 2015;115(1):21-26. doi: 10.1016/j. amjcard.2014.09.041.

24. Tonkin A, Barter P, Best J, et al. National Heart Foundation of Australia and the Cardiac Society of Australia and New Zealand: Position statement on lipid management 2005. Heart Lung Circ 2005;14(4):275-91.

25. Huffman MD, Xavier D, Perel P. Uses of polypills for cardiovascular disease and evidence to date. Lancet 2017;389(10073):1055-65. doi: 10.1016/ S0140-6736(17)30553-6. 


\section{Appendix 1. National Vascular Disease Alliance (NDVA) risk assessment algorithm - Clinical determinants of high cardiovascular disease (CVD) risk}

According to the risk assessment algorithm, adults with the following conditions do not require absolute CVD risk assessment using the Framingham risk equation because they are already known to be at clinically determined high risk of CVD:

- diabetes and age $>60$ years

- diabetes with microalbuminuria

- moderate or severe chronic kidney disease (persistent proteinuria or estimated glomerular filtration rate $<45 \mathrm{~mL} / \mathrm{min} / 1.73 \mathrm{~m}^{2}$ )

- a previous diagnosis of familial hypercholesterolaemia

- systolic blood pressure $\geq 180 \mathrm{mmHg}$ or diastolic blood pressure $\geq 110 \mathrm{mmHg}$

- serum total cholesterol $>7.5 \mathrm{mmol} / \mathrm{L}$

- Aboriginal and Torres Strait Islander adults aged $>74$ years.

Except for microalbuminuria and familial hypercholesterolaemia, all the above information was collected in the Busselton baby boomer survey participants. 